\title{
On Special Relativity and Temporal Illusions
}

Dimitria Electra Gatzia and Rex Ramsier

(Forthcoming in Erkenntnis)

According to metaphysical tensism, ${ }^{1}$ there is an objective, albeit ever-changing, present moment corresponding to our phenomenal experiences (Ludlow, 2012; Brogaard and Marlow, 2013). One of the principle objections to metaphysical tensism has been Einstein's argument from special relativity, according to which, given that the speed of light in vacuum is constant, there is no absolute simultaneity defined in terms of observations of light rays (Einstein, 1905).

In a recent paper entitled "Is the relativity of simultaneity a temporal illusion?" Brogaard and Marlow attempt to defuse the above objection. They construct a thought experiment, which they take to be analogous to Einstein's (1949), and use it to argue that Einstein's argument from special relativity does not threaten metaphysical tensism. In what follows, we show that the two thought experiments are not analogous and argue that, as a result, their argument fails to shield metaphysical tensism from the ramifications of special relativity.

Before we begin, some preliminary remarks are in order. Einstein deduced that there is no absolute simultaneity from the special theory of relativity - which states that physical laws should be the same in every inertial frame of reference, ${ }^{2}$ although they may vary across noninertial frames of reference-and the constancy of the speed of light detected in empty space, independent of the relative motion of its source or detector. To say that there is no absolute simultaneity is to say that whether two spatially separated events occur simultaneously is relative to an observer's frame of reference. In other words, it is impossible to say in an absolute sense whether two events that are separated in space occur at the same time. Einstein's claim is about absolute simultaneity, not the appearance of simultaneity. Events are simultaneous in the latter sense simply when our sensations of these events occur at the same moment. The relativity of simultaneity, however, is not about whether events are simultaneous in that sense; it is an issue that arises after we have corrected for the peculiarities of the appearance of simultaneity. Since there is no privileged inertial frame

1 The term "metaphysical tensism" was coined by Ludlow (2012) to suggest that tense is not a mere 2 Inertial frames of reference are those moving at constant velocity with respect to one another, i.e., they are neither speeding up, slowing down, changing direction, or rotating. 
relative to which one can judge absolute rest, Einstein concluded that simultaneity is relative to an inertial frame of reference. ${ }^{3}$

Let us now compare Einstein's thought experiment with that of Brogaard and Marlow's. In Einstein's experiment, M, a stationary observer located on a platform, sees ${ }^{4}$ two flashes of light simultaneously emitted from locations $\mathrm{A}$ and $\mathrm{B}$, equidistant to $\mathrm{M}$, corresponding to the beginning and endpoints of a moving train. At the same time, $\mathrm{M}^{*}$, who is located at the mid-point inside the moving train, sees the flash of light from A before he sees the flash of light from B because he is traveling towards A and away from B. Since there is no privileged inertial frame relative to which one can judge absolute rest, Einstein concluded that simultaneity is relative to an inertial frame of reference. ${ }^{1}$ In other words, even though $\mathrm{M}$ and $\mathrm{M}^{*}$ disagree about whether the two events occur simultaneously, neither observer is incorrect in their assessment.

Brogaard and Marlow attempt to undermine Einstein's claim that simultaneity is relative to an inertial frame of reference by constructing an experiment based on the principle of causality, which says that the cause must precede the effect (that it causes) as judged by all observers in inertial frames of reference. They ask us to assume that "a flash of light emitted from point A causes a flash to be emitted from point B" (Brogaard and Marlow, 2013: 638, emphasis added) and argue that since $\mathrm{A}$ and $\mathrm{B}$ are separated by two light-seconds, $\mathrm{M}$ who is located at the midpoint between $\mathrm{A}$ and $\mathrm{B}$ would conclude that B occurred two seconds after A. However $\mathrm{M}^{*}$, who is located on a moving train one light-second away from A when A occurs, traveling with a velocity of 0.5 light-seconds per second towards B, would see the two flashes of light as occurring simultaneously. In this scenario, although $\mathrm{M}$ and $\mathrm{M}^{*}$ disagree about whether the two events occur simultaneously, only one of them has made the correct assessment. Since $M^{*}$ fails to observe the cause and effect relation between the two events, it follows that it is $\mathrm{M}^{*}$ 's assessment that is incorrect.

Brogaard and Marlow assume that their thought experiment is analogous to Einstein's and argue that if $\mathrm{M}^{*}$ 's assessment is incorrect in their experiment, it stands to reason that $\mathrm{M}^{*}$ 's assessment can also be said to be incorrect in Einstein's experiment. Recall that in their experiment, $\mathrm{M}^{*}$ is "subject to a temporal illusion, owing to his or her increased

3 Unlike Einstein, his predecessors, Poincaré (1913) and Lorenz (1895) treated the aether as a preferred, albeit undetectable, frame of reference and distinguished between "true time" (in the aether) and "apparent times" for moving observers. 
velocity relative to the observer in the platform" (Brogaard and Marlow, 2013: 638). Given this, they argue that $M^{*}$ in Einstein's experiment must also be subject to a temporal illusion. If this is right, it follows that absolute simultaneity should be defined with respect to rest frames of reference extrapolated from real cases. But if simultaneity is defined in that way, Einstein's argument from special relativity ceases to pose a threat to metaphysical tensism.

Although Brogaard and Marlow consider various objections against their argument, they fail to notice that the two thought experiments are not analogous in the relevant respects. While Einstein's experiment assumes simultaneity of events followed by subsequently conflicting observations by two distinct observers, Brogaard and Marlow's experiment is described in such a way as to make simultaneity of events impossible. ${ }^{5}$ Recall that in their experiment, a flash of light emitted from point A causes a flash to be emitted from point B. On the basis of this description, Brogaard and Marlow go on to argue that $\mathrm{M}^{*}$, who is located on a moving train one light-second away from A when A occurs, traveling with a velocity of 0.5 light-seconds per second towards B, will conclude that $\mathrm{A}$ and B occurred simultaneously. Note, however, that this precarious conclusion is reached only because $\mathrm{M}^{*}$ is, by the very design of the thought experiment, exactly at $\mathrm{B}$ when the light from A reaches B. In other words, the inherent assumption made by Brogaard and Marlow, which they subsequently neglect in their analysis, is that when light from A reaches B, B emits light; so the "simultaneous" events (as observed by $\mathrm{M}^{*}$ ) are, by the very design of their experiment, in a relation of cause and effect! Note that $\mathrm{M}^{\wedge}$, a different stationary observer who happens to be at B, would see the same "simultaneity" as $\mathrm{M}^{*}$ in the Brogaard and Marlow scheme.

Of course, something has to transpire between the time light from $\mathrm{A}$ arrives at $\mathrm{B}$ and B emits its own light. So, if Brogaard and Mallow's moving observer M* (or our stationary observer $\left.\mathrm{M}^{\wedge}\right)$ is at $\mathrm{B}$ when the light from $\mathrm{A}$ arrives, and if $\mathrm{M}^{*}\left(\right.$ or $\left.\mathrm{M}^{\wedge}\right)$ had fast enough eyes (or some other type of fast detector with which to make observations), then $\mathrm{M}^{*}\left(\right.$ or $\mathrm{M}^{\wedge}$ ) would not see the events occurring simultaneously but instead as bearing the relation of cause and effect. Stationary observers anywhere else along the line between A and B would detect a time delay, as would any moving observers, with the lengths of the delays depending

${ }^{5}$ Note that in both experiments the events are the emission of light from A and B. The difference is that while these events are simultaneous by design in the rest frame of A and B in Einstein's setup, they stand in cause and effect relation in the rest frame of A and B in Brogaard and Marlow's setup. Observations by observers, moving or not, do not change the reality of either setup. 
on where they are when the light from A and B reaches them. It follows that no observer, in Brogaard and Marlow's setup, would experience the events as occurring simultaneously, since the light from A must be emitted and arrive at B before the light from B can be emitted.

Perhaps Brogaard and Marlow's thought experiment could be revised to avoid our criticism. Suppose, for example, that a detector which triggers flash B is placed in line between emitters A and B and connected to B via a perfectly straight, perfectly conductive electrical wire. In this setup, the flashes emitted from A and B could be imagined to arrive simultaneously at the moving observer $\mathrm{M}^{*}$ despite the fact that the light from $\mathrm{A}$ has not yet arrived at B. ${ }^{6}$ Although this scenario may seem to avoid the pitfalls of Brogaard and Marlow's thought experiment, it too fails to show that there is absolute simultaneity. A basic premise of the special theory of relativity is that no signal can travel faster than the speed of light in vacuum. No device or detection/transmission scheme, however perfect, can provide the information about where A's light is to B before A's light arrives at B.

We have argued that while Einstein's experiment is described in such a way as to make events simultaneous, Brogaard and Marlow's experiment is described in such a way as to make simultaneity impossible by forcing the events to be in a cause and effect relation. As we have shown, the two thought experiments are not analogous. It follows that in Einstein's experiment, $\mathrm{M}^{*}$ cannot be said to be mistaken based on Brogaard and Marlow's analysis. Brogaard and Marlow, therefore, fail to show that special relativity does not pose a threat to metaphysical tensism. Furthermore, any attempt to redescribe Brogaard and Marlow's thought experiment would be equally unsuccessful in rejecting the claim that there is no absolute simultaneity, since there can be no simultaneity in a situation designed to be cause and effect.

\section{References}

Brogaard, B. and Marlow, K. 2013. Is the relativity of simultaneity a temporal illusion? Analysis 73: 4: 635-642.

Einstein, A. 1905. On the electrodynamics of moving bodies. Annalen der Physik XVII: 891921.

Einstein, A. 1949. Autobiographical notes. In Philosopher-Scientist, ed. P.A. Schilpp, La Salle, IL: Open Court.

Lorentz, Hendrik Antoon (1895), Versuch einer Theorie der electrischen und optischen Erscheinungen

${ }^{6}$ We thank an anonymous referee for suggesting this scenario. 
in bewegten Körpern, Leiden: E.J. Brill.

Ludlow, P. 2012. Philosophy of Language, Oxford Handbook on Tense and Aspect. Oxford: Oxford University Press.

Poincaré, Henri (1913), "The Measure of Time", The foundations of science. New York: Science Press, pp. 222-234. 